\title{
Multiple Periodic Solutions for a Class of Second-Order Neutral Impulsive Functional Differential Equations
}

\author{
Jingli Xie, ${ }^{1}$ Zhiguo Luo, $^{2}$ and Yuhua Zeng ${ }^{3}$ \\ ${ }^{1}$ College of Mathematics and Statistics, Jishou University, Jishou, Hunan 416000, China \\ ${ }^{2}$ Department of Mathematics, Hunan Normal University, Changsha, Hunan 410081, China \\ ${ }^{3}$ Department of Mathematics, Hunan First Normal University, Changsha, Hunan 410205, China
}

Correspondence should be addressed to Jingli Xie; xiejingli721124@163.com

Received 29 August 2016; Revised 1 December 2016; Accepted 20 December 2016; Published 12 January 2017

Academic Editor: Olfa Boubaker

Copyright (c) 2017 Jingli Xie et al. This is an open access article distributed under the Creative Commons Attribution License, which permits unrestricted use, distribution, and reproduction in any medium, provided the original work is properly cited.

In this paper, we study a class of second-order neutral impulsive functional differential equations. Under certain conditions, we establish the existence of multiple periodic solutions by means of critical point theory and variational methods. We propose an example to illustrate the applicability of our result.

\section{Introduction and Main Results}

In this paper we consider a class of second-order neutral impulsive functional differential equations

$$
\begin{aligned}
& u^{\prime \prime}(t-\tau)-u(t-\tau) \\
& +\lambda f(t, u(t), u(t-\tau), u(t-2 \tau))=0, \\
& t \neq t_{j}, t \in J=[0,2 k \tau], \\
& \Delta u^{\prime}\left(t_{j}\right)=I_{j}\left(u\left(t_{j}\right)\right), \quad j=1,2, \ldots, l, \\
& u(0)-u(2 k \tau)=u^{\prime}(0)-u^{\prime}(2 k \tau)=0,
\end{aligned}
$$

where $f \in C\left(\mathbb{R}^{4}, \mathbb{R}\right), I_{j} \in C(\mathbb{R}, \mathbb{R})$, and $0=t_{0}<t_{1}<t_{2}<$ $\cdots<t_{l}<t_{l+1}=2 k \tau$. The operator $\Delta$ is defined as $\Delta u^{\prime}\left(t_{j}\right)=$ $u^{\prime}\left(t_{j}^{+}\right)-u^{\prime}\left(t_{j}^{-}\right)$, where $u^{\prime}\left(t_{j}^{+}\right)\left(u^{\prime}\left(t_{j}^{-}\right)\right)$denotes the right-hand (left-hand) limit of $u^{\prime}$ at $t_{j} . \lambda \in \mathbb{R}, \tau$ is a constant with $\tau>0$ and $k$ is a given positive integer.

The necessity to study delay differential equations is due to the fact that these equations are useful mathematical tools in modeling many real processes and phenomena studied in biology, medicine, chemistry, physics, engineering, economics, and so forth $[1,2]$.
On the other hand, impulsive differential equation not only is richer than the corresponding theory of differential equations but also represents a more natural framework for mathematical modeling of real world phenomena. People generally consider impulses in positions $u$ and $u^{\prime}$ for the second-order differential equation $u^{\prime \prime}=f\left(t, u, u^{\prime}\right)$. However it is well known that in the motion of spacecraft instantaneous impulses depend on the position which result in jump discontinuities in velocity, with no change in position.

Thus, it is more realistic to consider the case of combined effects: impulses and time delays. This motivates us to consider neutral impulsive functional differential system (1).

The existence of periodic solutions of delay differential equations has been focused on by many researchers [3-6]. Several available approaches to tackle them include Lyapunov method, Fourier analysis method, fixed point theory, and coincidence degree theory [7-10]. Recently, some researchers have studied the existence of solutions for delay differential equations via variational methods [11-13]. In recent years, some researchers, by using critical point theory, have studied the existence of solutions for boundary value problems, periodic solutions, and homoclinic orbits of impulsive differential systems [14-19].

In this paper, we aim to establish existence of multiple periodic solutions for the second-order neutral impulsive 
functional differential equation (1) by using critical point theory and variational methods.

For (1) with $I_{j}=0$, Shu and Xu [20] obtained the following periodic solutions result.

Theorem A. Assume that the following conditions are satisfied.

(H1) $\partial f\left(t, u_{1}, u_{2}, u_{3}\right) / \partial t \neq 0$.

(H2) There exists a function $F\left(t, u_{1}, u_{2}\right) \in C^{1}\left(\mathbb{R}^{3}, \mathbb{R}\right)$ such that

$\frac{\partial F\left(t, u_{1}, u_{2}\right)}{\partial u_{2}}+\frac{\partial F\left(t, u_{2}, u_{3}\right)}{\partial u_{2}}=f\left(t, u_{1}, u_{2}, u_{3}\right)$.

(H3) $F\left(t, u_{1}, u_{2}\right)$ is $\tau$-periodic in $t$.

(H4) $F$ satisfies $F\left(t,-u_{1},-u_{2}\right)=F\left(t, u_{1}, u_{2}\right)$ and $f\left(t,-u_{1}\right.$, $\left.-u_{2},-u_{3}\right)=-f\left(t, u_{1}, u_{2}, u_{3}\right)$.

(H5) $F\left(t, u_{1}, u_{2}\right)=0$ if and only if $\left(u_{1}, u_{2}\right)=0, \forall t \in[0, \tau]$.

(H6) $\lim _{|u| \rightarrow 0}\left(F\left(t, u_{1}, u_{2}\right) /|u|^{2}\right)=1$, where $|u|=\left(\left|u_{1}\right|^{2}+\right.$ $\left.\left|u_{2}\right|^{2}\right)^{1 / 2}, t \in[0, \tau]$.

(H7) There exists a constant $\alpha>0$ such that when $\left|u_{1}\right|^{2}+$ $\left|u_{2}\right|^{2}>\alpha^{2}, F\left(t, u_{1}, u_{2}\right)<0, t \in[0, \tau]$.

Moreover, if there exists an integer $m>0$ such that $\lambda$ satisfying

$$
\lambda>\frac{m^{2}\left(\pi^{2}+k^{2} \tau^{2}\right)}{4 k \tau^{2}},
$$

then the system

$$
\begin{aligned}
& u^{\prime \prime}(t-\tau)-u(t-\tau) \\
& \quad+\lambda f(t, u(t), u(t-\tau), u(t-2 \tau))=0, \\
& u(0)-u(2 k \tau)=u^{\prime}(0)-u^{\prime}(2 k \tau)=0
\end{aligned}
$$

possesses at least $2 m$ nonzero solutions with the period $2 k \tau$.

Our main result is stated as follows.

Theorem 1. Assume that (H1)-(H7) and the following condition are satisfied.

(H8) $I_{j}$ is odd about $u$, and there exists a constant $0 \leq D<1$ such that $\left|I_{j}(u)\right| \leq D|u|$, where $j=1,2, \ldots, l$.

Moreover, if there exists an integer $m>0$ such that

$$
\lambda>\frac{m^{2}\left(\pi^{2}+(1+D) k^{2} \tau^{2}\right)}{4 k^{2} \tau^{2}}
$$

then system (1) admits at least $2 m$ nonzero solutions with the period $2 k \tau$.

Clearly, when $I_{j}=0$, Theorem 1 generalizes Theorem A.

Note that the first equation of system (1) is equivalent to the following equation:

$$
\begin{aligned}
& u^{\prime \prime}(t-\tau)-u(t-\tau)+\lambda\left(F_{1}^{\prime}(t, u(t-\tau), u(t-2 \tau))\right. \\
& \left.\quad+F_{2}^{\prime}(t, u(t), u(t-\tau))\right)=0
\end{aligned}
$$

where $F_{1}^{\prime}(t, u(t-\tau), u(t-2 \tau))=\partial F(t, u(t-\tau), u(t-2 \tau)) / \partial u(t-$ $\tau)$ and $F_{2}^{\prime}(t, u(t), u(t-\tau))=\partial F(t, u(t), u(t-\tau)) / \partial u(t-\tau)$.
The rest of this paper is organized as follows. In Section 2, we present some preliminaries, which will be used to prove our main result. In Section 3 we prove our main result and provide an example to illustrate the applicability of our results.

\section{Some Preliminaries}

Let

$$
\begin{aligned}
& H_{2 k \tau}^{1}=\left\{u: \mathbb{R} \rightarrow \mathbb{R} \mid u, u^{\prime} \in L^{2}(([0,2 k \tau]), \mathbb{R}), u(0)\right. \\
& \left.\quad=u(2 k \tau), u^{\prime}(0)=u^{\prime}(2 k \tau)\right\} .
\end{aligned}
$$

Then $H_{2 k \tau}^{1}$ is a separable and reflexive Banach space and the inner product

$$
(u, v)=\int_{0}^{2 k \tau}\left(u^{\prime}(t) v^{\prime}(t)+u(t) v(t)\right) d t
$$

induces the norm

$$
\|u\|_{H_{2 k \tau}^{1}}=\left(\int_{0}^{2 k \tau}\left|u^{\prime}(t)\right|^{2}+|u(t)|^{2} d t\right)^{1 / 2} .
$$

Definition 2. A function $u \in H_{2 k \tau}^{1}$ is a solution of system (1) if the function $u$ satisfies system (1).

Define a functional $\varphi$ as

$$
\begin{aligned}
\varphi(u)= & \frac{1}{2} \int_{0}^{2 k \tau}\left(|u(t)|^{2}+\left|u^{\prime}(t)\right|^{2}\right) d t \\
& -\lambda \int_{0}^{2 k \tau} F(t, u(t), u(t-\tau)) d t \\
& +\sum_{j=1}^{l} \int_{0}^{u\left(t_{j}\right)} I_{j}(s) d s, \quad u \in H_{2 k \tau}^{1} .
\end{aligned}
$$

Then $\varphi$ is Fréchet differentiable at any $u \in H_{2 k \tau}^{1}$. For any $v \in$ $H_{2 k \tau}^{1}$, by a simple calculation, we have

$$
\begin{aligned}
& \varphi^{\prime}(u)(v)=\int_{0}^{2 k \tau}\left(u^{\prime}(t) v^{\prime}(t)+u(t) v(t)\right) d t \\
& -\lambda \int_{0}^{2 k \tau}\left(F_{1}^{\prime}(t, u(t), u(t-\tau)) v(t)\right. \\
& \left.+F_{2}^{\prime}(t, u(t), u(t-\tau)) v(t-\tau)\right) d t \\
& +\sum_{j=1}^{l} I_{j}\left(u\left(t_{j}\right)\right) v\left(t_{j}\right) .
\end{aligned}
$$

From (H3), we get

$$
\begin{gathered}
\varphi^{\prime}(u)(v)=\int_{0}^{2 k \tau}\left(-u^{\prime \prime}(t)+u(t)\right) v(t) d t \\
-\lambda \int_{0}^{2 k \tau}\left(F_{1}^{\prime}(t, u(t), u(t-\tau)) v(t)\right. \\
\left.+F_{2}^{\prime}(t, u(t+\tau), u(t)) v(t)\right) d t
\end{gathered}
$$


Therefore, the corresponding Euler equation of functional $\varphi$ is

$$
\begin{aligned}
& u^{\prime \prime}(t)-u(t) \\
& \quad+\lambda\left(F_{1}^{\prime}(t, u(t), u(t-\tau))+F_{2}^{\prime}(t, u(t+\tau), u(t))\right) \\
& =0 .
\end{aligned}
$$

Note that (6) is equivalent to system (13) and critical points of the functional $\varphi$ are classical $2 k \tau$-periodic solutions of system (1).

Definition 3 (see [21]). Let $E$ be a real reflexive Banach space, and

$$
\Sigma=\{A \mid A \subset E \backslash\{0\} \text { is closed, symmetric set }\} .
$$

Define $\gamma: \Sigma \rightarrow \mathbb{Z}^{+} \cup\{+\infty\}$ as follows:

$$
\gamma(A)= \begin{cases}\min \left\{n \in \mathbb{Z}^{+}: \text {there exists an odd continuous map } \varphi: A \rightarrow \mathbb{R}^{n} \backslash\{0\}\right\} \\ 0, & \text { if } A=\emptyset ; \\ +\infty, & \text { if there is no odd continuous map } \varphi: A \rightarrow \mathbb{R}^{n} \backslash\{0\} \text { for any } n \in \mathbb{Z}^{+}\end{cases}
$$

Then we say $\gamma$ is the genus of $\Sigma$.

Denote $i_{1}(\varphi)=\lim _{a \rightarrow-0} \gamma\left(\varphi_{a}\right)$ and $i_{2}(\varphi)=\lim _{a \rightarrow-\infty} \gamma\left(\varphi_{a}\right)$, where $\varphi_{a}=\{u \in E \mid \varphi(u) \leq a\}$.

Lemma 4 (see [22]). Let $E$ be a real Banach space and $\varphi \in$ $C^{1}(E, \mathbb{R})$ with $\varphi$ even functional and satisfying the PalaisSmale (PS) condition. Suppose $\varphi(0)=0$ and

(i) if there exist an $m$-dimensional subspace $X$ of $E$ and $a$ constant $r>0$ such that

$$
\sup _{u \in X \cap B_{r}} \varphi(u)<0
$$

where $B_{r}$ is an open ball of radius $r$ in $E$ centered at 0 , then we have $i_{1}(\varphi) \geq m$;

(ii) if there exists $j$-dimensional subspace $V$ of $E$ such that

$$
\inf _{u \in V^{\perp}} \varphi(u)>-\infty
$$

then we have $i_{2}(\varphi) \leq j$.

Moreover, if $m \geq j$, then $\varphi$ possesses at least $2(m-j)$ distinct critical points.

\section{Proof of Theorem 1 and an Example}

We apply Lemma 4 to finish the proof. Under assumption (H4), it is easy to see that if function $u$ is a solution of system (1), then function $-u$ is also a solution of system (1). Therefore, the solutions of system (1) are a set which is symmetric with respect to the origin in $H_{2 k \tau}^{1}$. It follows directly from (10), (H5), and (H8) that $\varphi$ is even in $u$ and $\varphi(0)=0$. The rest of the proof is divided into three steps.

Step 1. We show that the functional $\varphi$ satisfies assumption (ii) of Lemma 4.

It follows from (H7) that there exists a constant $M>0$ such that

$$
\max _{t \in \mathbb{R}} F(t, u(t), u(t-\tau)) \leq \max _{\left(t, u_{1}, u_{2}\right) \in \Omega} F\left(t, u_{1}, u_{2}\right) \leq M,
$$

where $\Omega=[0, \tau] \times[-\alpha, \alpha] \times[-\alpha, \alpha]$. Combining (10) and (18), we get

$$
\begin{aligned}
\varphi(u)= & \frac{1}{2} \int_{0}^{2 k \tau}\left(|u(t)|^{2}+\left|u^{\prime}(t)\right|^{2}\right) d t \\
& -\lambda \int_{0}^{2 k \tau} F(t, u(t), u(t-\tau)) d t \\
& +\sum_{j=1}^{l} \int_{0}^{u\left(t_{j}\right)} I_{j}(s) d s \\
\geq & \frac{1}{2} \int_{0}^{2 k \tau}\left|u^{\prime}(t)\right|^{2} d t+\frac{1-D}{2} \int_{0}^{2 k \tau}|u(t)|^{2} d t \\
& -2 \lambda M k \tau \geq \frac{1-D}{2}\|u\|_{H_{2 k \tau}^{1}}^{2}-2 \lambda M k \tau>-\infty,
\end{aligned}
$$

which implies that $\varphi$ is bounded from below. By condition (ii) of Lemma 4 , we have $i_{2}(\varphi)=0$.

Step 2. We show that the functional $\varphi$ satisfies the PS condition.

For any given sequence $\left\{u_{n}\right\} \in H_{2 k \tau}^{1}$ such that $\left\{\varphi\left(u_{n}\right)\right\}$ is bounded and $\lim _{n \rightarrow \infty} \varphi^{\prime}\left(u_{n}\right)=0$, there exists a constant $C_{1}$ such that

$$
\begin{aligned}
\left|\varphi\left(u_{n}\right)\right| & \leq C_{1}, \\
\left\|\varphi^{\prime}\left(u_{n}\right)\right\|_{\left(H_{2 k \tau}^{1}\right)^{*}} & \leq C_{1},
\end{aligned}
$$

$\forall n \in \mathbb{N}$,

where $\left(H_{2 k \tau}^{1}\right)^{*}$ is the dual space of $H_{2 k \tau}^{1}$.

Combining (19) and (20), we have

$$
\frac{1}{2}\|u\|_{H_{2 k \tau}^{1}}^{2} \leq C_{1}+2 \lambda M k \tau
$$

It follows that $\left\|u_{n}\right\|_{H_{2 k \tau}^{1}}$ is bounded. 
Since $H_{2 k \tau}^{1}$ is a reflexive Banach space, so we may extract a weakly convergent subsequence, for simplicity, we also note again by $\left\{u_{n}\right\}, u_{n} \rightarrow u$ in $H_{2 k \tau}^{1}$. So we have

$$
\begin{aligned}
& \int_{0}^{2 k \tau}\left(F_{1}^{\prime}\left(t, u_{n}(t), u_{n}(t-\tau)\right)-F_{1}^{\prime}(t, u(t), u(t-\tau))\right) \\
& \cdot\left(u_{n}(t)-u(t)\right) d t \longrightarrow 0, \\
& \int_{0}^{2 k \tau}\left(F_{2}^{\prime}\left(t, u_{n}(t), u_{n}(t-\tau)\right)-F_{2}^{\prime}(t, u(t), u(t-\tau))\right) \\
& \cdot\left(u_{n}(t-\tau)-u(t-\tau)\right) d t \longrightarrow 0, \\
& \sum_{j=1}^{l}\left(I_{j}\left(u_{n}\left(t_{j}\right)\right)-I_{j}\left(u\left(t_{j}\right)\right)\right)\left(u_{n}\left(t_{j}\right)-u\left(t_{j}\right)\right) \\
& \longrightarrow 0, \\
& u_{n}(t)-u(t) \longrightarrow 0 \text { as } n \longrightarrow \infty, t \in[0,2 k \tau] .
\end{aligned}
$$

Therefore, by (22), we have $\left\|u_{n}-u\right\|_{H_{2 k \tau}^{1}} \rightarrow 0$. Hence the functional $\varphi$ satisfies the PS condition.

Step 3. We show that the functional $\varphi$ satisfies assumption (i) of Lemma 4.

Let $\beta_{j}(t)=(k \tau / j \pi) \sin (j \pi / \kappa \tau) t, j=1,2, \ldots, m$. By calculations, we obtain

$$
\begin{aligned}
& \int_{0}^{2 k \tau}\left|\beta_{j}(t)\right|^{2} d t=\left(\frac{k \tau}{j \pi}\right)^{2} k \tau, \\
& \int_{0}^{2 k \tau}\left|\beta_{j}^{\prime}(t)\right|^{2} d t=k \tau .
\end{aligned}
$$

Define the $m$-dimensional linear subspace as follows:

$$
E_{m}=\operatorname{span}\left\{\beta_{1}(t), \beta_{2}(t), \ldots, \beta_{m}(t)\right\} .
$$

It is clear to see that $E_{m}$ is a symmetric set. Take $r>0$, when $u(t) \in E_{m} \cap S_{r}$, where $S_{r}$ denotes boundary of $B_{r}, u(t)$ has expansion $u(t)=\sum_{j=1}^{m} b_{j} \beta_{j}(t), b_{j} \in \mathbb{R}$, and

$$
\begin{aligned}
r^{2} & =\|u(t)\|_{H_{2 k \tau}^{1}}^{2}=\int_{0}^{2 k \tau}\left(\left|u^{\prime}(t)\right|^{2}+|u(t)|^{2}\right) d t \\
& \leq k \tau \sum_{j=1}^{m} b_{j}^{2}\left(1+\frac{k^{2} \tau^{2}}{j^{2} \pi^{2}}\right) .
\end{aligned}
$$

By (H6), for given $\varepsilon$ with $0<\varepsilon<\left(\lambda m^{2} / 4 k^{2} \tau^{2}\right)\left(4 k^{2} \tau^{2} /\right.$ $\left.m^{2}-\left(\pi^{2}+(1+D) k^{2} \tau^{2}\right) / \lambda\right)$, there exists $0<\delta<1$ such that when $\left(|u(t)|^{2}+|u(t-\tau)|^{2}\right)^{1 / 2}<\delta$, we have

$$
\begin{aligned}
& \lambda F(t, u(t), u(t-\tau)) \\
& \quad>(\lambda-\varepsilon)\left(|u(t)|^{2}+|u(t-\tau)|^{2}\right) .
\end{aligned}
$$

Combining (10), (25), and (26), when $u(t) \in E_{m} \cap S_{r}$, we have

$$
\begin{aligned}
\varphi(u) & =\frac{1}{2} \int_{0}^{2 k \tau}\left(|u(t)|^{2}+\left|u^{\prime}(t)\right|^{2}\right) d t \\
& -\lambda \int_{0}^{2 k \tau} F(t, u(t), u(t-\tau)) d t \\
& +\sum_{j=1}^{l} \int_{0}^{u\left(t_{j}\right)} I_{j}(s) d s \leq \frac{1}{2}\|u\|_{H_{2 k \tau}^{1}}^{2}-(\lambda-\varepsilon) \\
& \cdot \int_{0}^{2 k \tau}\left(|u(t)|^{2}+|u(t-\tau)|^{2}\right) d t+\frac{D}{2} \\
& \cdot \int_{0}^{2 k \tau}|u(t)|^{2} d t \leq \frac{k \tau}{2} \sum_{j=1}^{m} b_{j}^{2}\left(1+\frac{(1+D) k^{2} \tau^{2}}{j^{2} \pi^{2}}\right) \\
& -\frac{2(\lambda-\varepsilon) k^{2} \tau^{2}}{m^{2} \pi^{2}} k \tau \sum_{j=1}^{m} b_{j}^{2} \leq \frac{k \tau}{2 \pi^{2}} \\
& \cdot \sum_{j=1}^{m} b_{j}^{2}\left(\pi^{2}+(1+D) k^{2} \tau^{2}-\frac{4(\lambda-\varepsilon) k^{2} \tau^{2}}{m^{2}}\right) \\
& =\frac{\lambda k \tau}{2 \pi^{2}} \\
& \cdot \sum_{j=1}^{m} b_{j}^{2}\left(\frac{\pi^{2}+(1+D) k^{2} \tau^{2}}{\lambda}-\frac{4 k^{2} \tau^{2}}{m^{2}}+\varepsilon \frac{4 k^{2} \tau^{2}}{\lambda m^{2}}\right) \\
& <0 .
\end{aligned}
$$

Therefore $i_{1}(\varphi) \geq m$. Consequently, system (1) admits at least $2 m$ nonzero $2 k \tau$-periodic solutions.

We conclude this section with the following example.

Example 5. Consider (1) with

$$
\begin{gathered}
f(t, u(t), u(t-\tau), u(t-2 \tau)) \\
=4 u(t-\tau)-4\left(2+\cos \frac{2 \pi t}{\tau}\right) u(t-2 \tau) \\
\cdot\left(u^{2}(t)+2 u^{2}(t-\tau)+u^{2}(t-2 \tau)\right), \\
F\left(t, u_{1}, u_{2}\right)=u_{1}^{2}+u_{2}^{2}-\left(2+\cos \frac{2 \pi t}{\tau}\right)\left(u_{1}^{2}+u_{2}^{2}\right)^{2}, \\
I_{j}(u)=0.5 u .
\end{gathered}
$$

It is easy to see that $\partial f\left(t, u_{1}, u_{2}, u_{3}\right) / \partial t \neq 0$ and when $\left(u_{1}, u_{2}\right)=0, F\left(t, u_{1}, u_{2}\right)=0$; then (H1) and (H5) hold. Set $u_{1}=u(t), u_{2}=u(t-\tau), u_{3}=u(t-2 \tau)$, and then $\partial F(t, u(t), u(t-\tau)) / \partial u(t-\tau)+\partial F(t, u(t-\tau), u(t-2 \tau)) / \partial u(t-$ $\tau)=f(t, u(t), u(t-\tau), u(t-2 \tau))$. By a simple computation, we have $F\left(t+\tau, u_{1}, u_{2}\right)=F\left(t, u_{1}, u_{2}\right), F\left(t,-u_{1},-u_{2}\right)=$ $F\left(t, u_{1}, u_{2}\right)$, and $f\left(t,-u_{1},-u_{2},-u_{3}\right)=-f\left(t, u_{1}, u_{2}, u_{3}\right)$. So conditions (H2)-(H4) hold. Clearly, the conditions (H6)(H8) hold. Therefore system (1) admits at least $2 m$ nonzero solutions with the period $2 k \tau$. 


\section{Competing Interests}

The authors declare that there is no conflict of interests regarding the publication of this paper.

\section{Acknowledgments}

This work was partially supported by Hunan Provincial Natural Science Foundation of China (no. 2016JJ6122), National Natural Science Foundation of China (nos. 11661037 and 11471109), and Jishou University Doctor Science Foundation (no. jsdxxcfxbskyxm201504).

\section{References}

[1] J. K. Hale, Theory of Functional Differential Equations, Springer, New York, NY, USA, 1977.

[2] Y. Kuang, Delay differential equations with applications in population dynamics, Mathematics in Science and Engineering, Academic Press, Inc., Boston, MA, USA, 1993.

[3] W. Wang, P. Fergola, and C. Tenneriello, "Global attractivity of periodic solutions of population models," Journal of Mathematical Analysis and Applications, vol. 211, no. 2, pp. 498-511, 1997.

[4] R. Olach, "Positive periodic solutions of delay differential equations," Applied Mathematics Letters, vol. 26, no. 12, pp. 1141$1145,2013$.

[5] J. Yu and H. Xiao, "Multiple periodic solutions with minimal period 4 of the delay differential equation $\dot{x}(t)=-f(t, x(t-1))$," Journal of Differential Equations, vol. 254, no. 5, pp. 2158-2172, 2013.

[6] E. Serra, "Periodic solutions for some nonlinear differential equations of neutral type," Nonlinear Analysis: Theory, Methods \& Applications, vol. 17, no. 2, pp. 139-151, 1991.

[7] B. Yang, R. Ma, and C. Gao, "Positive periodic solutions of delayed differential equations," Applied Mathematics and Computation, vol. 218, no. 8, pp. 4538-4545, 2011.

[8] X. M. Li and X. P. Yuan, "Quasi-periodic solutions for perturbed autonomous delay differential equations," Journal of Differential Equations, vol. 252, no. 6, pp. 3752-3796, 2012.

[9] $\mathrm{K}$. Wu and X. Wu, "Multiplicity results of periodic solutions for a class of first order delay differential equations," Journal of Mathematical Analysis and Applications, vol. 390, no. 2, pp. 427438, 2012.

[10] J. Wu and Z. Wang, "Two periodic solutions of second-order neutral functional differential equations," Journal of Mathematical Analysis and Applications, vol. 329, no. 1, pp. 677-689, 2007.

[11] H. Xiao and Z. Guo, "Multiplicity and minimality of periodic solutions to delay differential system," Electronic Journal of Differential Equations, vol. 39, no. 115, pp. 1-12, 2014.

[12] X.-B. Shu, Y.-T. Xu, and L. H. Huang, "Infinite periodic solutions to a class of second-order Sturm-Liouville neutral differential equations," Nonlinear Analysis: Theory, Methods \& Applications, vol. 68 , no. 4, pp. 905-911, 2008.

[13] Z.-M. Guo and Y.-T. Xu, "Existence of periodic solutions to a class of second-order neutral differential difference equations," Acta Analysis Functionalis Applicata, vol. 5, no. 1, pp. 13-19, 2003.

[14] J. J. Nieto and D. O'Regan, "Variational approach to impulsive differential equations," Nonlinear Analysis: Real World Applications, vol. 10, no. 2, pp. 680-690, 2009.

[15] Z. Zhang and R. Yuan, "An application of variational methods to Dirichlet boundary value problem with impulses," Nonlinear Analysis. Real World Applications, vol. 11, no. 1, pp. 155-162, 2010.
[16] L. Bai and B. X. Dai, "An application of variational method to a class of Dirichlet boundary value problems with impulsive effects," Journal of the Franklin Institute, vol. 348, no. 9, pp. 26072624, 2011.

[17] Y. Tian, J. Wang, and W. Ge, "Variational methods to mixed boundary value problem for impulsive differential equations with a parameter," Taiwanese Journal of Mathematics, vol. 13, no. 4, pp. 1353-1370, 2009.

[18] J. Xie and Z. Luo, "Subharmonic solutions with prescribed minimal period of an impulsive forced pendulum equation," Applied Mathematics Letters, vol. 52, pp. 169-175, 2016.

[19] J. Xie and Z. Luo, "Homoclinic orbits for Hamiltonian systems induced by impulses," Mathematical Methods in the Applied Sciences, vol. 39, no. 9, pp. 2239-2250, 2016.

[20] X. B. Shu and Y. T. Xu, "Multiple periodic solutions to a class of second-order functional differential equations of mixed type," Acta Mathematicae Applicatae Sinica, vol. 29, no. 5, pp. 821-831, 2006.

[21] J. Mawhin and M. Willem, Critical Point Theory and Hamiltonian Systems, Springer, Berlin, Germany, 1989.

[22] C. Q. Ching, Critical Point Theory and Its Applications, Shanghai Scientific and Technical Publishers, Shanghai, China, 1986. 


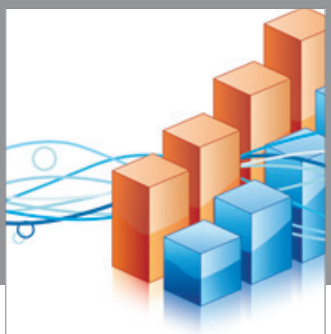

Advances in

Operations Research

vatem alat4

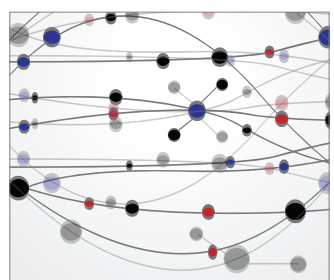

\section{The Scientific} World Journal
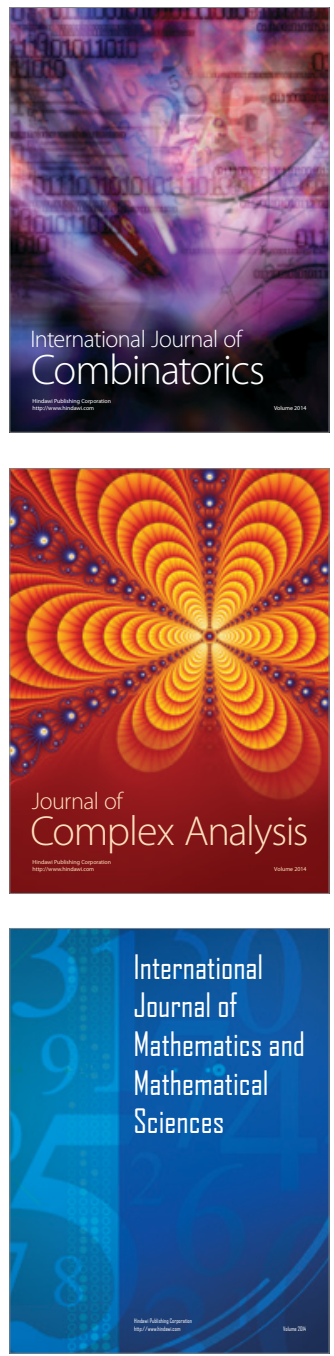
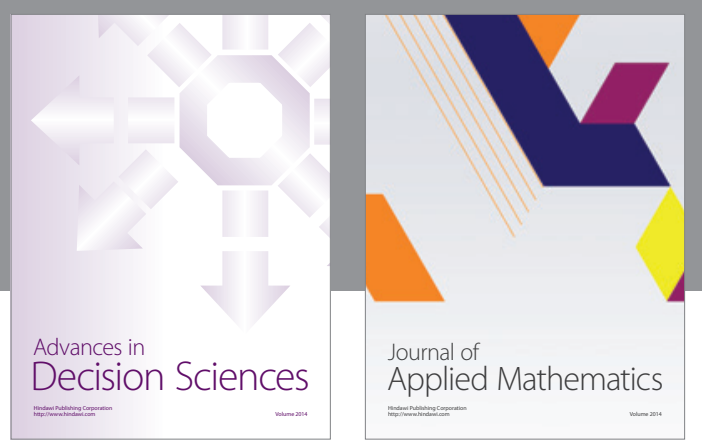

Algebra

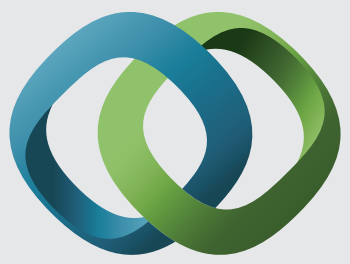

\section{Hindawi}

Submit your manuscripts at

https://www.hindawi.com
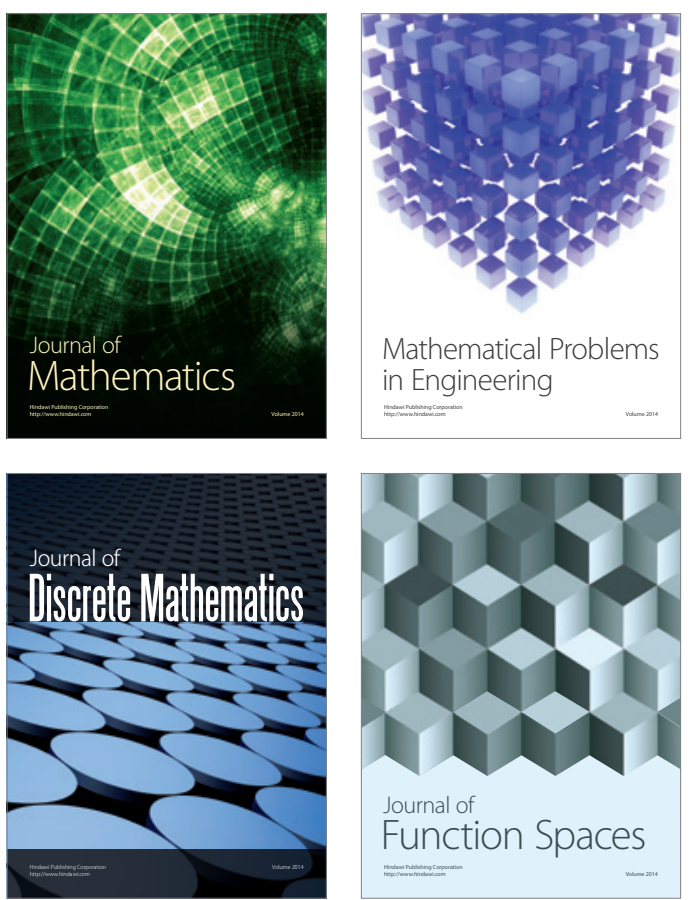

Mathematical Problems in Engineering
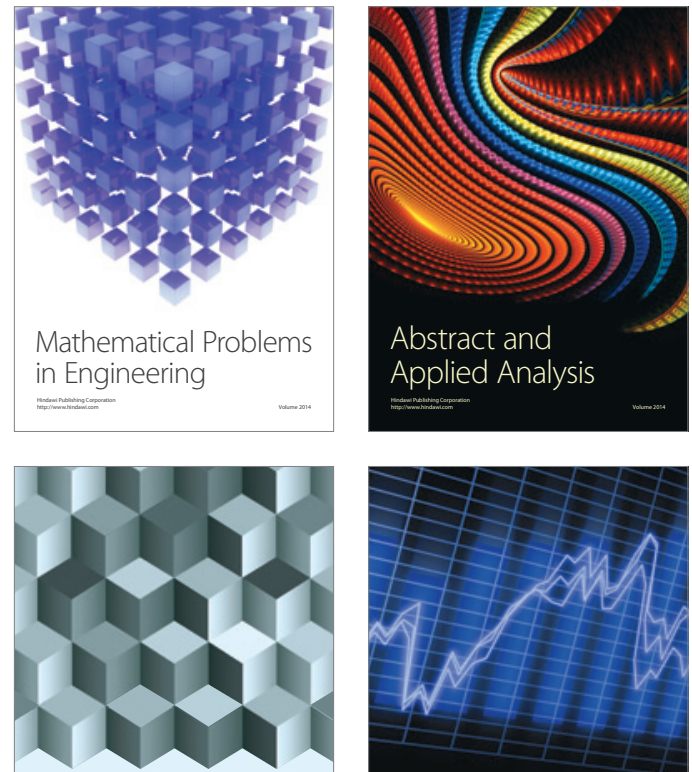

Journal of

Function Spaces

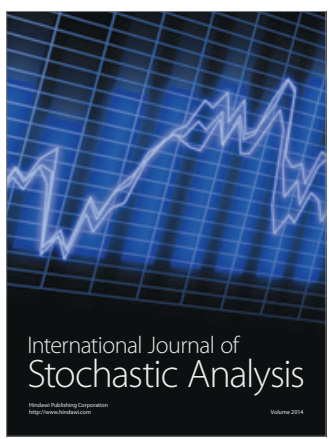

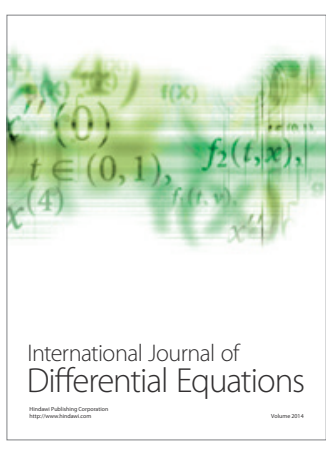
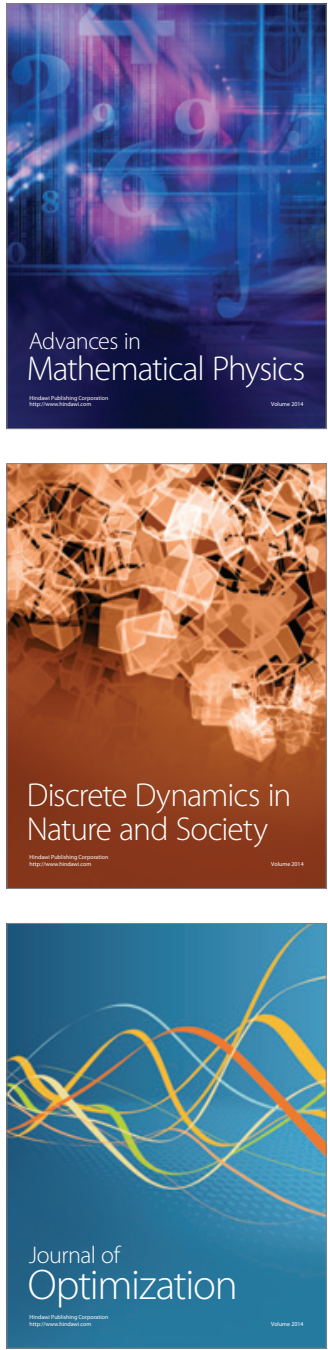